\title{
Pulmonary Vein Thrombosis in a Patient With Multiple Myeloma on Treatment With Lenalidomide
}

\author{
Kristin M. Stawiarski ${ }^{\mathrm{a}, \mathrm{d}}$, Gourav Patil ${ }^{\mathrm{b}}$, David Witt ${ }^{\mathrm{c}}$, Ari Pollack ${ }^{\mathrm{a}}$
}

\begin{abstract}
Multiple myeloma (MM) poses inherent risk of thrombosis that can be amplified by the use of immunomodulator therapy. We present a patient with $\mathrm{MM}$ who was being treated with lenalidomide and dexamethasone when he developed progressive dyspnea on exertion consistent with a left lower pulmonary vein thrombosis (PVT) despite use of prophylactic aspirin. The PVT was not initially seen on standard computed tomography angiogram pulmonary embolism protocol but was seen on 192-slice multidetector computed tomography angiogram for assessment of coronary calcifications 8 months later. Subsequent treatment with full dose rivaroxaban resulted in full clot resolution and symptom improvement. PVT has not been previously reported with lenalidomide therapy and may not be a forefront differential diagnosis. In such cases, a multi-modality diagnostic approach may be beneficial. Consideration should be given to escalating venous thromboembolism prophylaxis to full dose anticoagulation during increased prothrombotic windows, such as the time of treatment initiation or dose adjustments, in low bleeding risk patients.
\end{abstract}

Keywords: Thrombosis; Multiple myeloma; Immunotherapy; Prevention

\section{Introduction}

Pulmonary vein thrombosis (PVT) is a rare life-threatening condition seen in the setting of malignancy, lung surgery, or atrial fibrillation [1]. However, its clinical presentation is highly variable making diagnosis challenging. Suspicion should remain high in cases where additional prothrombotic risk fac-

Manuscript submitted April 19, 2021, accepted May 10, 2021

Published online May 14, 2021

aSection of Cardiovascular Medicine, Yale New Haven Health Bridgeport Hospital, Bridgeport, CT, USA

bDepartment of Internal Medicine, Griffin Hospital, Derby, CT, USA

'Hematology Disease Associated Research Team, Yale Smilow Cancer Hospital, New Haven, CT, USA

${ }^{d}$ Corresponding Author: Kristin M. Stawiarski, Yale New Haven Health Bridgeport Hospital, 267 Grant Street, Bridgeport, CT 06610, USA.

Email:kmstawiarski@gmail.com

doi: https://doi.org/10.14740/wjon1384 tors are present. We describe a patient with multiple myeloma (MM) who was being treated with lenalidomide and dexamethasone when he developed a left lower PVT diagnosed on cycle 10 of therapy.

\section{Case Report}

A 67-year-old man with past medical history significant for hypertension, hyperlipidemia, benign prostatic hypertrophy, and sleep apnea presented with increasing fatigue and worsening pancytopenia over the past 8 months. Subsequent bone marrow biopsy revealed $13 \%$ plasma cells and a high kappa/ lambda light chain ration $>8$. In the absence of hypercalcemia, renal injury, significant anemia, or bone lytic lesions on positron emission tomography and computed tomography (CT), the patient was diagnosed with smoldering myeloma. He was entered into the phase III DETER-SMM (Daratumumab to Enhance Therapeutic Effectiveness of Revlimid in Smoldering Myeloma) clinical trial (clinicaltrials.gov, NCT03937635) for treatment of high-risk smoldering myeloma and was randomized to the lenalidomide and dexamethasone combination therapy control arm. His dose regimen comprised of 24 treatment cycles including: lenalidomide $25 \mathrm{mg}$ daily from day 1 to day 21 with 7-day rest time (cycles 1 - 24), and dexamethasone dose of $40 \mathrm{mg}$ on days 1, 8, 15 and 22 (cycles 1 - 6) followed by $20 \mathrm{mg}$ on days $1,8,15$ and 22 between cycles 7 and 12 . He was also start on aspirin $325 \mathrm{mg}$ for venous thromboembolism (VTE) prophylaxis.

Within 3 weeks of starting chemotherapy, patient developed sudden onset exertional dyspnea. Electrocardiogram (ECG) showed normal sinus rhythm with voltage criteria for left ventricular hypertrophy and left atrial abnormality with negative troponin. Urgent pulmonary computed tomography angiogram (CTA) was negative for pulmonary embolism. Further, stress echocardiogram revealed dynamic $3-4 \mathrm{~mm} \mathrm{ST}$ depressions in the inferolateral leads without any echocardiographic findings of ischemia. However, patient was unable to reach the target heart rate due to shortness of breath and drop in oxygen saturation to $92 \%$. Moderate concentric left ventricular hypertrophy was identified with a resting gradient across the left ventricular outflow tract (LVOT) of $26 \mathrm{~mm} \mathrm{Hg}$ that increased to $48 \mathrm{~mm} \mathrm{Hg}$ at peak exercise and $54 \mathrm{~mm} \mathrm{Hg}$ with Valsalva maneuver. Cardiac magnetic resonance imaging (MRI) had normal perfusion with no delayed myocardial enhancement and left ventricular ejection fraction of $80 \%$ with 


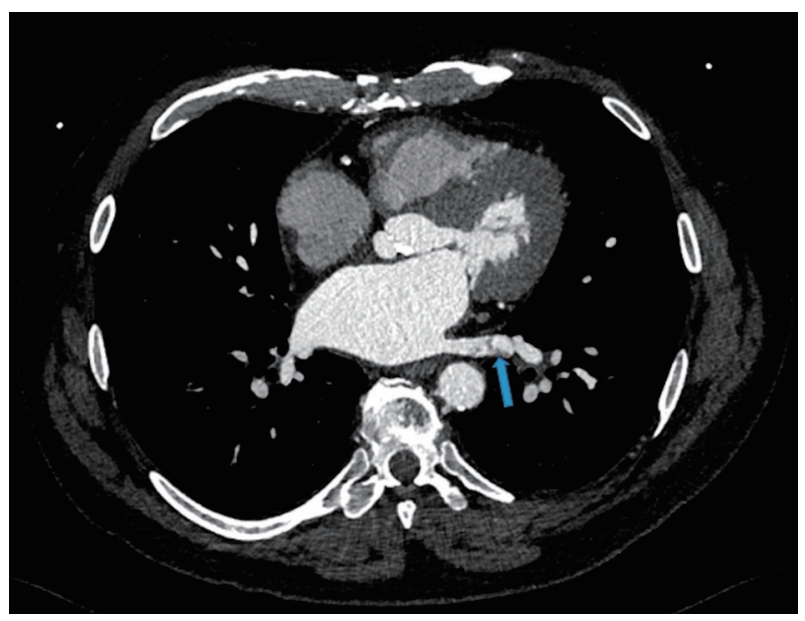

Figure 1. Left lower pulmonary vein thrombosis. Transverse view of chest via a gated 192-slice multidetector computed tomography angiogram revealed a lower pulmonary vein thrombosis (arrow).

turbulent flow in the LVOT. There was no suggestion of cardiac amyloidosis. His LVOT obstruction was thought to be the source of his symptoms and metoprolol was added to his medication regimen along with a decrease in lisinopril. Chemotherapy was continued as planned.

However, due to persistent neutropenia and rising liver enzymes, lenalidomide was lowered to $15 \mathrm{mg}$ at cycle 5 . This was further reduced to $10 \mathrm{mg}$ by cycle 6 and $5 \mathrm{mg}$ by cycle 10 for similar. During the 10th cycle, patient had a syncopal episode with worsening shortness of breath. Due to previously suboptimal stress test, coronary CTA was performed along with fractional flow reserve (FFR). CT-FFR showed diffuse atherosclerotic disease in left anterior descending (0.79) and left circumflex coronary artery $(0.72)$ that did not warrant intervention. However, a thrombus in the left lower pulmonary vein was identified (Fig. 1). He was started on rivaroxaban $20 \mathrm{mg}$. Lenalidomide, dexamethasone and aspirin were discontinued. A 2-week follow-up transesophageal echocardiogram showed resolution of the pulmonary vein clot. Repeat CTA performed 2 months after initial diagnosis also confirmed clot resolution (Fig. 2). He had dramatic improvement in his dyspnea and remains off treatment for MM with plans to continue with rivaroxaban indefinitely given patient's underlying malignancy.

\section{Discussion}

PVT is a rare entity that often presents with nonspecific symptoms including dyspnea, syncope, or chest pain [1]. Most commonly PVT occurs in patients with atrial fibrillation or malignancy. Post-operatively, it is seen in up to $17.9 \%$ of those receiving left upper lobectomy and $15 \%$ of lung transplant recipients carrying with it a $38 \%$ mortality at 90 days [1]. To our knowledge, there have been no reported cases of PVT with lenalidomide treatment.

Lenalidomide is an immunomodulator with anti-inflammatory and anti-angiogenic properties that further increase

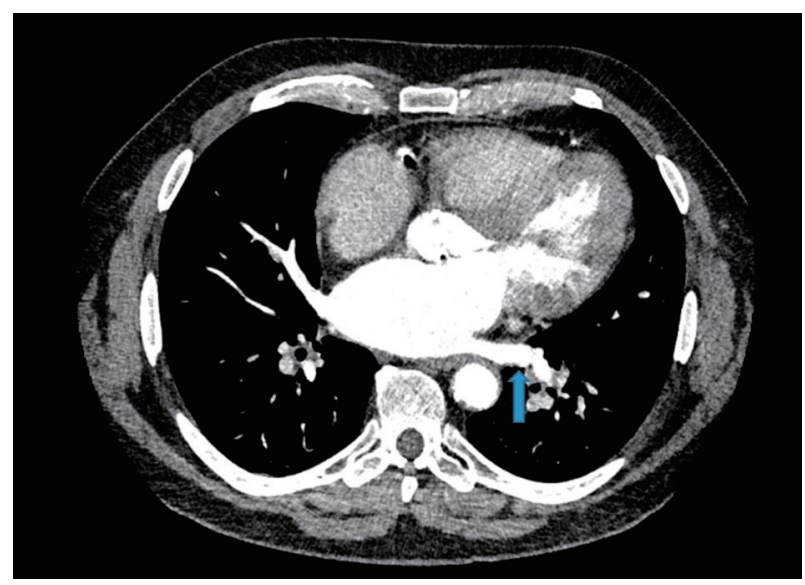

Figure 2. Resolution of left lower pulmonary vein thrombus. Repeat computed tomography angiogram revealed clot resolution after treatment with rivaroxaban. Similar transverse view of the chest is shown with blue arrow marking the previously seen thrombus location.

the risk of VTE in already prothrombotic MM patients [2]. This risk is amplified three-fold when lenalidomide is combined with steroid therapy with an incidence of VTE as high as $4 \%$ [2]. Current guidelines suggest prophylaxis with either low-molecular weight heparin (LMWH) or low-dose aspirin for MM patients on anti-angiogenesis agents based on a tiered risk factor approach [3]. LMWH or full dose warfarin with a target international ration of $2-3$ is recommended for patients with $\geq 2$ risk factors for VTE including advanced age, history of VTE, recent surgery, degree of disease burden, or combination steroid therapy [4]. Direct oral anticoagulants are not currently advised; however, there is an ongoing prospective randomized controlled trial of apixaban $2.5 \mathrm{mg}$ twice daily versus to evaluate the rate of VTE in this population over 6 months [5].

With its diverse clinical presentation, the diagnosis of PVT relies heavily on imaging studies. Chest X-ray findings are often nonspecific requiring advanced imaging modalities [1]. Chest CTAs, although the typical test of choice in clinical practice, visualize arterial anatomy often leaving true filling venous defects versus mixing artifacts up to question. Our patient had a CTA performed at the onset of symptoms approximately 3 weeks into MM therapy which did not reveal a PVT. It is possible that the PVT could have been present at that time but not detected until he underwent a gated 192-slice multidetector computed tomography (MDCT) angiogram for assessment of coronary calcifications 8 months later. Similarly, pulmonary venous phased protocols or 64-slide MDCT scans have been used as diagnostic alternatives [1]. Although transesophageal echocardiogram offers the ability to evaluate posterior cardiac structures including the pulmonary vein, CT offers a further advantage in cases of mural PVT as pulmonary air may obscure the vessel wall [6]. Likewise, MRI can help distinguish between a bland versus a tumor thrombus in the pulmonary vein given persistently high signal intensity on the first and second echoes of spin-echo sequences in bland thrombus [7]. No gold standard diagnostic test or follow-up imaging protocol exists. Instead, imaging modalities should be tailored 
to the clinical scenario with considerations for invasiveness of test, radiation and contrast exposure as well as timely availability.

Systemic anticoagulation is the agreed upon therapy once PVT is diagnosed [1]. However, the types and duration of anticoagulation are not yet defined [1]. Successful treatment with both oral vitamin $\mathrm{K}$ antagonists and heparin (unfractionated and low molecular weight) has been reported [1]. To our knowledge, four cases of PVT post lung resection have been reportedly treated with rivaroxaban therapy [8, 9]. Three of these patients had brain infarcts as a complication of PVT and saw successful resolution of the thrombus within 1 month [9]. However, a 68-year-old man developed an idiopathic right upper PVT with extension into the left atrium while on rivaroxaban $20 \mathrm{mg}$ daily for known atrial fibrillation [8]. After resection, anticoagulation was changed to dabigatran with resolution of clot at 1 year [8]. Likewise, an earlier reported case of PVT found on 64-slice MDCT also showed clot resolution with dabigatran as patient declined warfarin therapy due to its narrow therapeutic range requiring frequent drug monitoring [10]. Rivaroxaban was chosen for our patient given ease of administration with once daily dosing regimen. Although the duration of anticoagulation is not standardized, lifelong anticoagulation was chosen for our patient given that his MM is a persistent non-modifiable risk factor.

Our case adds PVT to the differential diagnosis for patients with new shortness of breath on immunomodulator therapy. With increased awareness, further investigations into the frequency, diagnostic techniques and treatment of PVT during lenalidomide therapy will be feasible.

\section{Learning points}

1) The standard CTA pulmonary embolism protocol may not offer optimal screening for PVT and instead a multi-modality approach may be necessary when suspected.

2) Regardless, consideration should be given to escalating VTE prophylaxis to full dose anticoagulation during increased prothrombotic windows, such as the time of treatment initiation or dose adjustments, in low bleeding risk patients.

3) Rivaroxaban may be an effective therapeutic choice for patients with PVT.

\section{Acknowledgments}

None to declare.

\section{Financial Disclosure}

None to declare.

\section{Conflict of Interest}

None to declare.

\section{Informed Consent}

Informed consent was obtained.

\section{Author Contributions}

All authors have contributed equally to the intellectual content of this report. Each author has reviewed the final version of the manuscript and approved it for publication. KS and GP wrote the manuscript and reviewed the literature. AP and DW were involved in the diagnosis and management of the patient and supervised the manuscript creation including making final edits. AP gave the suggestion to write the manuscript.

\section{Data Availability}

The authors declare that data supporting the findings of this study are available within the article.

\section{Abbreviations}

MDCT: multidetector computed tomography; ECG: electrocardiogram; MM: multiple myeloma; PVT: pulmonary vein thrombosis; CTA: computed tomography angiogram; LVOT: left ventricular outflow tract; VTE: venous thromboembolism

\section{References}

1. Chaaya G, Vishnubhotla P. Pulmonary vein thrombosis: a recent systematic review. Cureus. 2017;9(1):e993.

2. Carrier M, Le Gal G, Tay J, Wu C, Lee AY. Rates of venous thromboembolism in multiple myeloma patients undergoing immunomodulatory therapy with thalidomide or lenalidomide: a systematic review and meta-analysis. J Thromb Haemost. 2011;9(4):653-663.

3. Lyman GH, Bohlke K, Khorana AA, Kuderer NM, Lee AY, Arcelus JI, Balaban EP, et al. Venous thromboembolism prophylaxis and treatment in patients with cancer: american society of clinical oncology clinical practice guideline update 2014. J Clin Oncol. 2015;33(6):654656.

4. Palumbo A, Rajkumar SV, Dimopoulos MA, Richardson PG, San Miguel J, Barlogie B, Harousseau J, et al. Prevention of thalidomide- and lenalidomide-associated thrombosis in myeloma. Leukemia. 2008;22(2):414-423.

5. Cornell RF, Goldhaber SZ, Engelhardt BG, Moslehi J, Jagasia M, Patton D, Harrell S, et al. Apixaban for primary prevention of venous thromboembolism in patients with multiple myeloma receiving immunomodulatory therapy. Front Oncol. 2019;9:45.

6. Takeuchi H. A 64-slice multi-detector CT scan could evaluate the change of the left atrial appendage thrombi of the atrial fibrillation patient, which was reduced by warfarin therapy. BMJ Case Rep. 2011;2011:bcr1120092491. 
7. Selvidge SD, Gavant ML. Idiopathic pulmonary vein thrombosis: detection by $\mathrm{CT}$ and MR imaging. AJR Am J Roentgenol. 1999;172(6):1639-1641.

8. Lebel K, Tan S, Parent MC, El-Hamamsy I, Couture C, Laroussi L. An unusual presentation of a cardiac thrombus. Can J Cardiol. 2019;35(10):1420.E1-1420.E3.

9. Amemiya T, Shono T, Yamagami K, Takagishi S, Toma
H, Kobarai T, Eguchi T, et al. Usefulness of oral Xa inhibitor for management of ischemic stroke associated with thrombosis in the pulmonary vein stump after lung resection. J Stroke Cerebrovasc Dis. 2019;28(11):104321.

10. Takeuchi H. Floating thrombus in the left upper pulmonary vein dissolved by dabigatran. BMJ Case Rep. 2013;2013:bcr2013200836. 